\title{
First implantation of the CE marked transcatheter sapien 3 ultra-valve in pulmonary position
}

\author{
Tanase $\mathrm{D}^{1 *}$, Georgiev $\mathbf{S}^{1}$, Kasel $\mathbf{M}^{2}$ and Ewert $\mathbf{P}^{1}$ \\ ${ }^{1}$ Department of Pediatric Cardiology and Congenital Heart Defects, German Herat Centre Munich, Germany \\ ${ }^{2}$ Department of Cardiology, German Heart Centre Munich, Germany
}

\begin{abstract}
The Edwards Sapien transcatheter valves have been used for more than ten years in pulmonary position. One concern of these valves was that they had to be crimped distally to the inflation balloon and adjusted in the inferior caval vein. The Sapien 3 ultra-recently received CE mark for the aortic position. In contrast to the former Sapien transcatheter valves, as the Sapien 3 and Sapien XT, this one can be crimped directly on the inflation balloon, facilitating the procedure. We report on the first successful implantation of the Sapien 3 ultra in pulmonary position.
\end{abstract}

\section{Introduction}

Transcatheter pulmonary valve implantation was first performed in 2000 by Phillip Bonhoefer with a precursor of the Melody valve (Melody valve, Medtronic, Minneapolis, MN, USA) [1]. For about 10 years the Edwards Sapien valve, originally designed for the aortic position, has been used in its different modifications in pulmonic position [2, 3]. One concern in these valves has been that the valve has to be mounted in the opposite direction onto the delivery system and then it has to be positioned within the inferior caval vein on the correct place of the dilation balloon. This must be done by pulling the balloon into the crimped valve against the flow direction, i.e. against the orientation of the valve leaflets. The Edwards Sapien 3 ultra can be crimped on its own delivery system without this positioning maneuver inside the patient's body. We report on the first implication of this CE marked valve in the pulmonic position.

\section{Case Report}

A 19-year-old young woman, born with pulmonary atresia, ventricular septal defect (VSD) and multifocal aortopulmonary collaterals, was palliated by unifocalisation of the collaterals and a central aortopulmonary shunt in her second year of life. Ten months later, "corrective" surgery was performed by closure of the VSD, removement of the shunt and connection of the right ventricle to the pulmonary vessels with a Hancock conduit (Hancock Bioprosthetic Valved Conduit, Medtronic, Minneapolis, MN, USA). At the age of 8 years, the first conduit exchange was performed with a $22 \mathrm{~mm}$ VenPro heterograft (VenPro, Venpro Corporation, Irvine, CA, USA). Four years later, the graft showed severe regurgitation and the patient's exercise tolerance dropped. Thus, a transcatheter Melody valve was implanted. Now, seven years later, the Melody valve had developed a systolic peak invasive pressure gradient of up to $35 \mathrm{mmHg}$ with a right ventricular systolic pressure of $70 \mathrm{mmHg}$, which reflected three quarters of systemic pressure, and the patient had noted a decline in exercise tolerance with a $\mathrm{VO}_{2} \max$ of $18.2 \mathrm{ml} / \mathrm{kg} / \mathrm{min}$ ( $56 \%$ of the reference value). Therefore, the valve was dilated with a $26 \mathrm{~mm}$ Atlas balloon (ATLAS PTA Dilatation Catheter, Bard Peripheral Vascular, Inc, AZ,
USA). There was considerable recoil, but the pressure gradient dropped to $15 \mathrm{mmHg}$. The valve, however, was severely regurgitant. In order to overcome the recoil and to abolish the regurgitation, a $26 \mathrm{~mm}$ Edwards Sapien 3 ultra was implanted without any additional pre-stenting or dilation (for inflation of the balloon and the deployment of the Sapien 3 Ultra see the movie file in the supplementary material). The valve was crimped with the inflow to the handle of the delivery system in an otherwise regular way. The insertion through the groin was easily performed through the new improved 14F AXELA sheath (Edwards Lifescience Irvine CA, USA) and the advancement of the delivery system to the target region was very smooth as was the implantation of the valve itself (Figure 1).

Post-implant angiography revealed no regurgitation of the symmetrically configured valve and a catheter pullback showed a residual gradient of about $8 \mathrm{mmHg}$ across the valve with the right ventricular systolic pressure of $40 \mathrm{mmHg}$. Fluoroscopy time was $21.3 \mathrm{~min}$.

\section{Discussion}

The introduction of transcatheter valves for the pulmonary position has amplified the interventional possibilities in the treatment of patients with congenital heart disease enormously [4]. The Edwards Sapien transcatheter valves are especially helpful in large outflow tracts. In comparison to the Melody valve, the Sapien had a more complex crimping and delivery procedure. With the new Edwards Sapien 3 ultra, the implantation procedure is considerably simplified. Especially since the valve is already crimped in its final delivery position and does not need to be pulled against the flow direction within the inferior caval vein, the implantation procedure is much straighter forward. After insertion of the crimped valve the only procedural step before implanting the valve is the retraction of the pusher which is facilitated

${ }^{\star}$ Correspondence to: Daniel Tanase, German Heart Centre Munich, Lazarettstrasse 36, 80363 Munich, Germany, E-mail: tanase@dhm.mhn.de

Key words: percutaneous valve, sapien 3 ultra, pulmonary valve

Received: July 20, 2019; Accepted: August 05, 2019; Published: August 08, 2019 


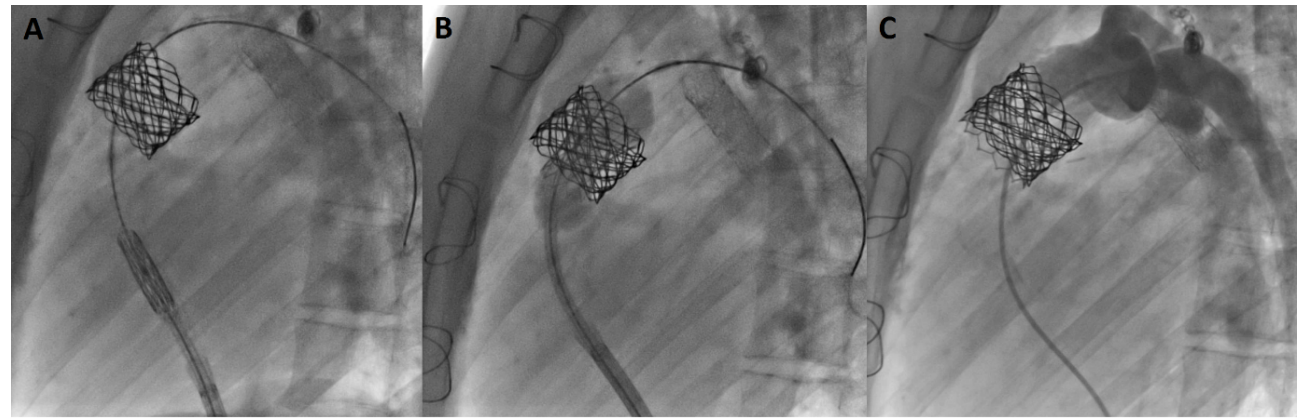

Figure 1. Implantation of the valve. Note that the valve is already crimped on the balloon during the advancement, indicated by the radiopaque marker (A). The Sapien3 ultra is positioned correctly during the inflation with the proximal end slightly overlapping the previously implanted Melody valve (B). A complete movie of the implantation is available as supplementary material. The final frame shows the result of the procedure without regurgitation of contrast agent into the right ventricle (C).

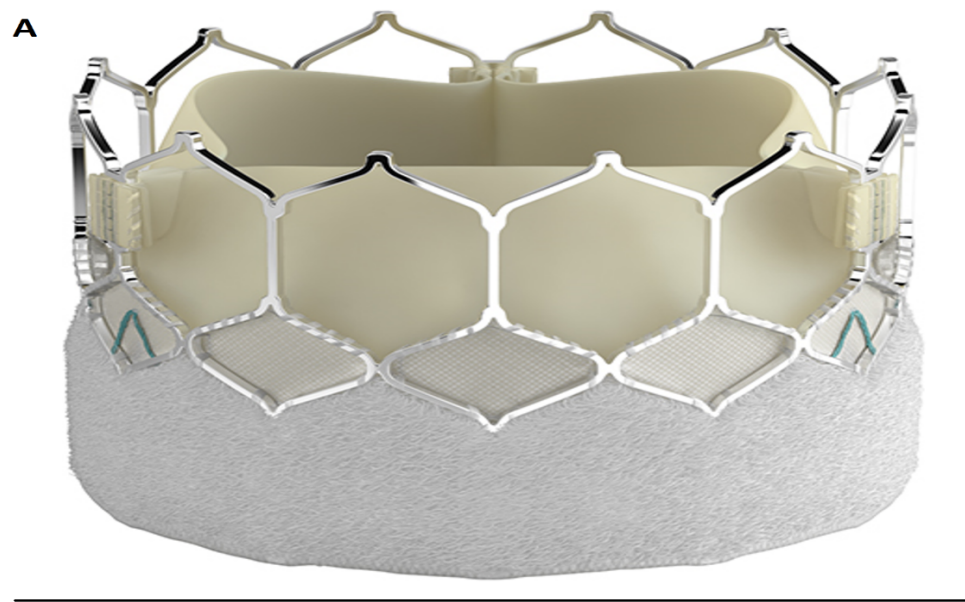

$\mathbf{B}$

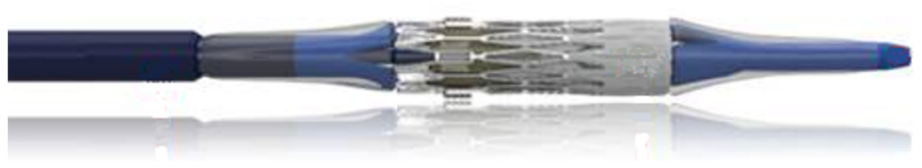

Figure 2. The Sapien 3 ultra-valve (A) and its delivery system (B). In the figure the Sapien 3 ultra is crimped on the balloon with the outer skirt forward, as necessary for the aortic position. In the presented patient the valve has been crimped reverse for the pulmonic position.

by a well-defined turning of a corresponding screw at the handle of the delivery system (Figure 2). It is noteworthy that the implantation sheath has been improved as well. While in the former sheath the expansion of the elastic sheath by the cimped valve resulted in a longitudinal relatively sharp edge, the improved actual version has an additional elastic layer around the surface so that even after reaching the level of the right atrium the surface of the sheath remains without any edge, minimizing the risk for vessel damage.

\section{Conclusion}

The introduction of the Edwards Sapien 3 ultra has the potential to be an effective further step to optimize transcatheter valve implantation in pulmonary position.

\section{References}

1. Bonhoeffer P, Boudjemline Y, Saliba Z, Merckx J, Aggoun Y, et al. (2000) Percutaneous replacement of pulmonary valve in a right-ventricle to pulmonary-artery prosthetic conduit with valve dysfunction. Lancet 356: 1403-1405. [Crossref]

2. Kenny D, Rhodes JF, Fleming GA, Kar S, Zahn EM, et al. (2018) 3-Year Outcomes of the Edwards SAPIEN Transcatheter Heart Valve for Conduit Failure in the Pulmonary Position from the COMPASSION Multicenter Clinical Trial. JACC Cardiovasc Interv 11: 1920-1929. [Crossref]

3. Ewert P, Horlick E, Berger F (2011) First implantation of the CE-marked transcatheter Sapien pulmonic valve in Europe. Clin Res Cardiol 100: 85-87. [Crossref]

4. Eicken A, Ewert P, Hager A, Peters B, Fratz S, et al. (2011) Percutaneous pulmonary valve implantation: two-centre experience with more than 100 patients. Eur Heart $J$ 32: 1260-1265. [Crossref]

Copyright: (C2019 Tanase D. This is an open-access article distributed under the terms of the Creative Commons Attribution License, which permits unrestricted use, distribution, and reproduction in any medium, provided the original author and source are credited. 